simultaneously in any hospital, but Dr. Humphry had three at one time under his care, all undertaken for laryngitis, and all doing well. Excision of the knee is, as might be expected from the numerous cases reported from this hospital, a not infrequent operation. The horseshoe incision is made, and the patella generally removed. Temperature-charts are in regular use with all severe cases.

Enough has been said to show that Addenbrooke's Hospital presents in itself great capabilities as a clinical school, and that it affords a valuable field of instruction and experience for the medical students of the university.

\section{SUMMARY OF \\ PAPERS READ AT THE MEDICAL SECTION OF THE FRENOH ASSOCIATION FOR THE ADVANCEMENT OF SCIENCE.}

\author{
BY HENRY BLANC, M.D., F.R.G.S., \\ SURGHON-MAJOR, H.M. INDIAN ABYYY.
}

The Medical Section of the French Association was presided over by Professor Verneuil, the eminent surgeon of La Pitié.

Dr. Ollier, well known to the English medical public by his remarkable labours on the Reproduction of the Bones, read a paper "On the various Chirurgical means by which the Growth of Bones is Increased or Arrested." Dr. Ollier made many experiments on animals in order to ascertain the process under which the growth of bones takes place. He believes, with Flourens, that the intermediate cartilage is an important agent of their evolution. Some years ago he removed, in young animals, the intermediate cartilage of some of the long bones, and he obtained the following results:-Immediate arrest of the growth of the bone at the extremity from which the intermediate cartilage had been removed, the bone still growing on at the opposite end. Intense irritation of the cartilage gave results similar to those obtained by its removal. Dr. Ollier has also observed that osteitis, situate in close proximity to the cartilages, causes the bones to cease growing at the diseased extremity. If, on the other hand, a long bone be irritated in parts remote from its cartilages, it will increase in length; if a bone, still growing, be irritated in any part of its shaft, either by application of caustics or other means, the bone will increase in length from a sixteenth to a twelfth of its total length.

Some very convincing and well-prepared specimens of the results obtained on animals were exhibited by Dr. Ollier, and fully proved the correctness of his assertions.

Dr. Ollier has applied these physiological facts to practical surgery; he has operated on superficial bones, and employed, as an irritant, the Vienna paste, which he applies so as to reach the bone after the destruction of the integuments. On several occasions he has obtained a considerable increase in the length of the bones. He quoted the following case :-A young girl was admitted into his wards at the Hôtel Dieu of Lyons suffering from osteitis of the tibia adjoining the cartilage. On recovery this bone was found to be twenty-four millimetres shorter than the sound one. To the anterior surface of the shorter tibia Dr. Ollier made an application of Vienna paste. Some time afterwards be repeated the cauterisation, this time using the "pâte de Cauquoin." The bone slightly exfoliated. Five weeks afterwards a slight increase in length was apparent, and three months and a half from the date of the operation the bone had gained in length thirteen millimetres.

Where a permanent irritation is required, it is necessar to repeat the application of caustic; the action of the caustic should be carried to the bone itself. The fibula need not be interfered with. The elongation of the tibia will cause the smaller bone to be dragged down with it; a partial dislocation takes place at the upper extremity, but the foot remains straight.

To arrest the growth of a long bone the cartilage should be partly destroyed, but without penetration in to the joint. This arrest of growth is also obtained by the removal of a small piece of the cartilage; after these operations the wound must be carefully closed and the limb immobilised.

Dr. Ollier has performed this operation twice successfully. On one occasion a purulent osteitis of the radius near its intermediate cartilage had caused the bone to cease to grow, whilst the ulna, still increasing in length, had deviated the hand inwardly. All orthopædic means having failed, Dr. Ollier destroyed part of the cartilage of the ulna in order to arrest its growth; a few months afterwards the hand was already straighter, and ultimately it recovered its normal direction, as we were able to ascertain from plaster casts of the forearm taken before the operation and after recovery.

Dr. Ollier remarked on the numerous applications to surgery of this power to control the growth of bones; not only had experiments made on animals proved the correctness of his views, but clinical observations had demonstrated their practical value, and testified to their curative power in certain diseases of the bony structures of man.

Professor Chauveau, the able physiologist, to whom we are indebted for our present knowledge of the intricate nature of some viruses, made an extremely interesting communication "On the Transmission of Tuberculosis through the Digestive Organs." He called attention to this question as one of great importance to the public health. During several years he has made numerous experiments on animals. He administered tubercular matter with their food, and he arrived at the conclusion that the bovine race only is susceptible of contracting tuberculosis.

Professor Chauveau has repeatedly administered tubercular matter to calves; the animals continue in good health for about six weeks, but soon after that lapse of time they begin to lose flesh and ail. All calves to which tubercular matter was administered with their food presented on postmortem examination evident and numerous signs of tuberculosis, some in an acute, some in a chronic form; the disease affects generally the lungs, and pervades to a variable extent the glandular system.

In order to show these facts to the members of the Association, Professor Chauveau prepared the following experiment. He purchased four calves. Two of them were born the same day and on the same farm, the other two were of about the same age and size, and came from farms situate in the vicinity of the first. They were brought to the Lyons Veterinary School on the 17th of June; until the 25th of that month they were all fed alike on milk and barley-meal. The animals thrived well. On the 25th he selected the two strongest calves, and administered to them, mixed with water, fifteen grammes of tubercular matter taken from the lungs, bronchi, and glands of a cow that had died of tuberculosis. This was again administered to both of them and in the same way on the $26 \mathrm{th}$ and on the 30 th of June; on the 6th of July a fourth dose was given; this time the tubercular matter was mixed with flour to a pasty consistence, and made to be swallowed as boluses. During five weeks the calves continued in good health. At the date of the meeting (22nd August) one of the calves only was ailing; he had lost flesh and swallowed his food with some difficulty. The two calves not subjected to the ingestion of tubercular matter seemed in perfect health.

At Professor Chauveau's request a committee was appointed by the Medical Section to make a post-mortem examination of the calves. In their report the committee stated that they had found in the calves to which tubercular matter had been administered extensive infiltration of tubercles in the lungs and in many of the glands. In one of these they found deposits of tubercles in the cervical glands and a large tubercular ulceration in the pharynx.

The two calves to which no tubercular matter had been administered were also examined. Here also tuberculosis was present, but in a much more limited and less advanced condition. This fact, which at first sight seemed to disprove the views held by the learned professor, were found on investigation to strongly corroborate them, and afforded a still greater proof of the high contagious power of tubercular matter. Professor Chauveau had been absent for some weeks, and the feeding of the calves had been entrusted to one of his subordinates; the calves had been kept apart, as directed, but fed all of them from the same bottle. It is probable that some tubercular matter from the pharyngeal ulceration of one of the calves adhered to the mouth of the bottle, and thus inoculated the healthy calves with the 
disease. In order to test the value of this explanation and ascertain if tuberculosis were not a common disease among calves in that neighbourhood, the lungs and glands of ten calves were carefully examined, and in none of them the faintest traces of tuberculosis could anywhere be found.

(To be continued.)

\section{foretgin oblemings.}

\section{INFLUENCE OF VARIATIONS OF PRESSURE ON VEGETABLES.}

M. BERT having studied the influence of variations of pressure on animals, communicated to the Académie des Sciences (July 16th, 1873) the results of his inquiries upon the influence of variations of pressure upon vegetables. Germination, he finds, takes place more slowly in proportion to the lowness of the pressure, being altogether stopped when it diminishes to from four to ten centimetres, though grains thus rendered inactive do not die. The arrest of the germinating process is here evidently due to stoppage of the oxidation requisite for the development of the embryo, or, in other words, to the too feeble tension of the oxygen. Seeds placed under a pressure of two or three atmospheres seem to germinate somewhat more rapidly and vigorously than those in air of ordinary pressure, but if the pressure be augmented to four or five atmospheres, germination is obviously interfered with, especially in seeds the albumen of which is farinaceous. Finally, in air at a much higher pressure seeds are killed, even when germination has begun This appears to be due exclusively to the too great tension of the oxygen, and is much more evident in farinaceous seeds than in others. If the changes that have taken place in the compressed air be compared with those in air at normal pressure, the consumption of oxygen will be found to be much less in the compressed air. Thus in one case, in four days, 10 grammes of barley germinating in ordinary air consumed 225 cubic centimetres of oxygen, whilst a similar quantity placed in air superoxygenated to the extent of 2.5 atmospheres, corresponding to a tension of 11 atmo spheres of air, consumed only 136 cubic centimetres. 'Ihus it appears that an excessive tension of oxygen retards germination.

\section{INFLAMMATION OF THE THORACIC CANAL.}

Dr. Chouppe, of Paris, has just published a thesis in which he has collected five cases of the above rare and, as zet, not well known affection. The five cases collected by Dr. Chouppe are the only ones hitherto placed on record. In three of the cases the inflammation was secondary, and, in two, primary. In order to distinguish this latter form from the former, Dr. Chouppe has conducted a series of experiments on animals. The points brought out by the two cases and by the author's experiments are very interesting. In both cases the inflamed thoracic canal had thrown a quantity of pus into the general circulation through the subclavian vein, and it might be said that the patients had had a sort of pjæmia through an internal course; yet, during life, typhoid fever, meningitis, and articular rheumatism were successively suspected, whilst purulent infection was not even thought of. Another point of interest was the obliteration of the subclavian vein in one of the cases; it could only, however, have occurred during a late period of the disease, as symptoms of pus in the blood had been previously observed. Notwitbstanding the great importance of the thoracic canal, as conveying all the chyle produced by digestion, and the most part of ly mph, no disturbance in the digestive organs or in the functions of nutrition was observed. Moreover, Dr. Chouppe stated the existence of pulmonary infarctus, caused, according to him, by the pus producing embolisms. The other symptoms were redness of the surface in various parts, inflammation of the sheaths of tendons, and of several joints. In both primary cases the onset of the disease was sudden in one case it came on with violent pain in the stomach and intense fear ; in the other, with a violent and protracted fit of rigor. In the two cases these symptoms were followed by high fever, prostration, loss of strength and flesh.

Dr. Chouppe has arrived at the following conclusions on the important question which he has investigated:Primary inflammation of the thoracic canal does exist; it is attended by general symptoms of purulent infection and by local symptoms; it cannot be diagnosed as yet during life by the belp of known symptoms, but its presence may be suspected ; it is likely that it has been sometimes overlooked, and that it occurs occasionally.

\section{UNILATERAL PULMONARY APOPLEXY DEPENDENT} ON CEREBRAT HAMORRHAGE,

In the number just published (for August) of the Archives Médicales there is an interesting paper on the above by Dr. Ollivier. The author has observed three unmistakable cases of the dependence of pulmonary apoplexy on hæmorrhage of the brain, not to reck on other cases in which the connexion of the two sorts of lesion was less evident. In all three cases the pulmonary apoplexy occupied the same lung as the paralysed side, and, consequently, that opposite to the cerebral lesion. In all three cases it was the left cerebral hemisphere that had suffered hæmorrhage. From these observations, and others of a similar character, Dr. Ollivier concludes that, under the influence of cerebral hæmorrhage, various lesions may develop in the lung corresponding to hemiplegia-namely, more or less intense and extensive congestion, considerable sanguineous effusions under the pleura, or else true apoplexy. This apoplexy may be either diffuse or circumscribed. Again, there may exist hypostatic pneumonia. It seems that the considerable extent of the hæmorrhage, and the contact of the blood with the pia mater and arachnoid, are influential in the production of unilateral pulmonary apoplexy or congestion. It is likely that other affections of the brain (tumours, softening, \&c.) may, under certain circumstances of situation and extent, produce the same lung lesions. These lung lesion: may supervene rapidly after the attack of cerebral apoplexy (in one case dulness on percussion was observed nine hours after). It is likely that, according to Dr. Brown-Séquard, the connexion of the head and lung lesions is through the vaso-motors, and that the lung apoplexy is due to simultaneous contraction of the veins and arteries pushing forcibly the blood into the capillaries. Dr. Brown-Séquard, it may be added, upholds the view that the vaso-motors of the thoracic and abdominal viscera do not come from the medulla. oblongata, but from the pons Varolii and adjoining parts.

\section{VARIX OF THE SUPERIOR LONGITUDINAL SINUS}

\section{OF THE DURA MATER.}

This case is recorded by Dr. Meschede in Virchow's Archiv, Band 57, Heft 3-4, 1873 . The patient had been an epileptic sufferer from the age of six years, had always shown a weak mind, and became insane towards the age of twenty-six. He had often had cerebral congestion, with rise of temperature in the head and abundant sweats, especially in the situation of the vertex, from which veritable vapour was often seen to rise. He died from meningeal apoplexy at the age of thirty-seven. At the post-mortem enormous distension of the veins of the pia mater was discovered, and in the situation of the vertex there was found a tumour of the size of a white bean, full of coagulated blood, lodged in a cavity bored out in the cranium, and communicating manifestly with the superior longitudinal sinus. It was a true varix.

PROGRESSIVE MUSCULAR HYPERMEGALTA.

By the above name Professor Orsi designates a case of Duchenne's "myo-sclerosic paralysis"-a term which he likes less than his own for defining the pathogeny of the disease. The case is described in a number of La Gazetta Medica Lombardia. Dr. Orsi recommends for the treatment of the disease the following:-Faradisation of the diseased muscles, passive exercise, nitrogenous food, and a mixture of carbonate of iron and nux vomica.

\section{OCCLUSION OF THE EUSTACHIAN TUBE.}

Dr. Rudinger, in Monatschrift der Ohrenheitkunde, No. 3 , 1873, after a series of experiments carried on upon himself, has arrived at the conclusion that the Eustachian tube is habitually closed. When it is accidentally opened through a contraction or cramp of the dilating muscle of the tube, the voice has a peculiar sound for the person who is speaking, and resumes its natural sound only when the tube is again closed. 